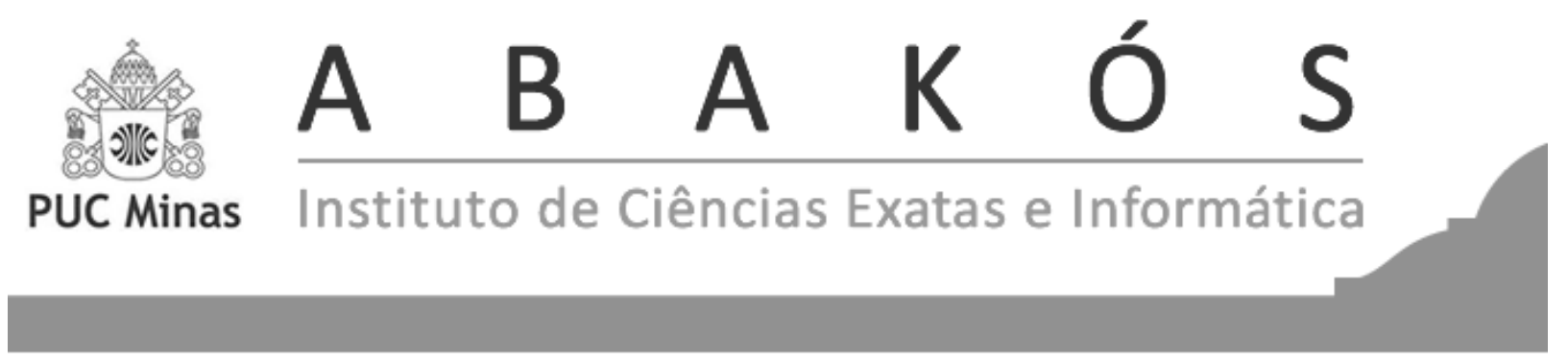

\title{
Desenvolvimento de Aplicativos Web Com R e Shiny: inovações no ensino de Estatística*
}

Development of Web Applications with R and Shiny: innovations in teaching statistics

\author{
Andréa Cristina Konrath ${ }^{1}$ \\ Silvio Aparecido da Silva ${ }^{2}$ \\ Elisa Henning ${ }^{3}$ \\ Luciane Mulazani dos Santos ${ }^{4}$ \\ Rodrigo Gabriel de Miranda ${ }^{5}$ \\ Robert Wayne Samohyl ${ }^{6}$
}

\begin{abstract}
Resumo
A utilização de objetos de aprendizagem com interfaces digitais, interativas e dinâmicas é uma alternativa que pode ser inovadora no ensino de conceitos de Estatística e na realização de suas análises, uma vez que possibilita tipos de visualização e interatividade que, em grande parte das vezes, não são alcançados no ambiente lápis e papel. Neste artigo, apresenta-se uma discussão a respeito das características e das potencialidades do pacote Shiny do software R para desenvolver aplicativos web voltados ao ensino e à pesquisa, no âmbito da Estatística estudada no Ensino Superior. O Shiny é um pacote desenvolvido para usuários do software R, que é um ambiente para computação Estatística. Com o Shiny, podem-se criar aplicações interativas em linguagem HTML, de forma simples e intuitiva, sem necessidade de conhecimento de outras linguagens de programação além da linguagem R. Neste trabalho, foi desenvolvido uma aplicação por meio do pacote Shiny, utilizando os resultados do ENEM no Estado de Santa Catarina.
\end{abstract}

Palavras-chave: Ensino. Software livre. Shiny.

\footnotetext{
* Submetido em 10/05/2017 - Aceito em 27/03/2018

${ }^{1}$ Departamento de Informática e Estatística, Universidade Federal de Santa Catarina, Brasil- andrea.ck@ufsc.br

${ }^{2}$ Doutorando do Programa de Pós-Graduação em Engenharia de Produção, Universidade Federal de Santa Catarina, Brasil-silvioest@gmail.com

${ }^{3}$ Departamento de Matemática, Universidade do Estado de Santa Catarina, Brasil- elisa.henning@ gmail.com

${ }^{4}$ Departamento de Matemática, Universidade do Estado de Santa Catarina, Brasil- lucianemulazani@gmail.com

${ }^{5}$ Centro de Ciências da Administração e Socioeconômicas, Universidade do Estado de Santa Catarina, Brasilrgabrieldemiranda@yahoo.com.br

${ }^{6}$ Membro do Núcleo de Normalização e Qualimetria, Universidade Federal de Santa Catarina, Brasil-

samohyl@yahoo.com
} 


\begin{abstract}
The use of interactive and dynamic interfaces is an alternative that can be innovative in the teaching of statistical concepts and in the performance of statistical analysis, as it enables views and manipulations that are hardly achieved in a paper and pencil environment. In this paper, a discussion of the characteristics and potential of the R Package Shiny for the development of web applications dedicated to teaching and research is presented. Shiny is a package designed for users of the software $\mathrm{R}$, an environment for statistical computing. With it, one can create interactive applications in HTML, in a simple and intuitive way, without the need for knowledge of other programming languages, besides the $\mathrm{R}$ language. In this project, an application was developed through the Shiny package, using the ENEM results of the Santa Catarina State.
\end{abstract}

Keywords: Teaching. Free Software. Shiny. 


\section{INTRODUÇÃO}

As Tecnologias de Informação e Comunicação (TIC) estão cada vez mais presentes no dia a dia da sociedade, estimulando e viabilizando a utilização de software em diferentes atividades e de objetos de aprendizagem em diferentes contextos do ensino formal e informal. No Ensino Superior, por exemplo, há certo consenso entre professores que ministram disciplinas como Estatística, sobre a necessidade do desenvolvimento de práticas docentes apoiadas em algum tipo de TIC com o objetivo de diminuir a escrita de cálculos apenas no ambiente lápis/papel ou giz/quadro e, também, de permitir aos alunos, de maneira dinâmica e interativa, o acesso a conjuntos de dados de casos práticos e situações reais (SCHUYTEN; THAS, 2007; GOULD, 2010). Na busca de soluções para resolver questões como essa, constrói-se um novo direcionamento para o Ensino Superior, que possibilita aos educadores desenvolverem e utilizarem alternativas educacionais capazes de integrar as novidades da ciência e da tecnologia com o engajamento dos alunos de modo a tornar mais atrativo e efetivo os processos de ensino e aprendizagem (MINUTO et al., 2015).

Os aplicativos web, utilizados no ambiente da Internet, por serem dinâmicos e interativos, se mostram como objetos de aprendizagem interessantes e eficientes. Para o trabalho com a Estatística, há uma interface gráfica do software R, chamada RStudio, cuja possibilidade de manipulações e de mudanças de diferentes recursos permite a criação de ambientes ímpares e atrativos para os acadêmicos e pesquisadores, contemplando tanto o ensino como a pesquisa (MINUTO et al., 2015).

O R (R CORE TEAM, 2017) é um software livre de código aberto. O conceito de software livre surgiu na década de 80 para se contrapor aos softwares pagos (FERNANDES et al., 2012). O objetivo era permitir o desenvolvimento de sistemas que fossem totalmente livres, que pudessem ser modificados, transformados, acrescidos de funções de acordo com as necessidades dos usuários ou das organizações. Aqui, é importante comentar sobre a diferença entre software livre e de código aberto. O software livre se configura quase como um movimento social, indo além da preocupação com o código fonte de um programa, incluindo questões filosóficas que envolvem consequências econômicas, políticas e sociais (CAMPOS; CAZARINI, 2005; CORREA; SPINOLA, 2015). Já o termo código aberto se refere a uma metodologia de desenvolvimento e, como tal, tem preocupação com a garantia de disponibilidade do código fonte e suas modificações de modo que o software livre se configure como um movimento social (CAMPOS; CAZARINI, 2005; CORREA; SPINOLA, 2015).

Nesse contexto, mais do que um software, o R é uma linguagem e um ambiente de desenvolvimento integrado para computação estatística. Relacionados a ele, há uma interface gráfica amigável chamada RStudio e um pacote de uso direto pela Internet, o Shiny.

Neste artigo, pretende-se descrever e discutir as potencialidades do pacote Shiny do software $\mathrm{R}$ para o desenvolvimento de aplicativos web voltados ao ensino e à pesquisa. Inicialmente, apresenta-se o R e a interface RStudio. Na sequência, está a descrição do Shiny e a discussão sobre suas potencialidades de uso no Ensino Superior. 


\section{R E RSTUDIO}

O R é uma linguagem e um ambiente para computação estatística no formato de projeto de software livre de código aberto (open source), ou seja, pode ser utilizado sem custos de licença. Há versões para Windows, MacOS, GNU/Linux e Unix. Pode ser baixado da Internet pelo site do Comprehensive R Archive Network (CRAN) - Rede Completa de Arquivos do R - no endereço: http://www.r-project.org. Possui uma extensa coleção de pacotes adicionais, também gratuitos. É resultado de um esforço colaborativo mundial de vários pesquisadores, tais como estatísticos e engenheiros de software, que constituem o Core Team, equipe responsável pela avaliação e atualizações semestrais de novos pacotes (KATAOKA et al., 2008).

Além do trabalho com as funções padrões do R, na área da Estatística, é possível utilizar pacotes desenvolvidos pela comunidade para resolver problemas avançados relacionados a várias áreas de conhecimento. Desse modo, o R é uma plataforma multidisciplinar voltado para análises estatísticas, porém, com diversos pacotes de aplicações que podem contribuir em áreas como Engenharia, Economia, Biologia, Ciência da Saúde e Ciências Sociais.

Originalmente, o R é um programa conduzido por meio de digitação de linhas de comando, o que pode dificultar a sua operacionalização por pessoas que não possuem conhecimentos de programação. Alguns autores mencionam que pode ser difícil aprender a utilizá-lo, principalmente em nível introdutório (BAIER; NEUWIRTH, 2007; FOX, 2007; VERZANI, 2008). Por outro lado, é importante ressaltar que o ambiente de programação pode ser considerado um dos pontos positivos do $\mathrm{R}$, uma vez que se parte do princípio de que cada usuário, acadêmico ou pesquisador pode ser um programador (KONRATH et al., 2013).

Para aprimorar a sua usabilidade, foram desenvolvidas interfaces gráficas, entre elas o RStudio (RACINE, 2012). O RStudio é um ambiente de desenvolvimento integrado (Integrated Development Environment - IDE) que reúne diversas funcionalidades, tornando mais amigáveis aos usuários a importação de dados, visualização de comandos, funções, resultados e gráficos, além da geração de documentos (HENNING et al., 2016). O download do RStudio está disponível em http://rstudio.org/ e o mesmo pode ser feito para vários sistemas operacionais. A tela principal do RStudio é disposta em 4 janelas de visualização, como mostrado na Figura 1. No canto superior, à esquerda, a janela Source é a área de trabalho onde são disponibilizados os códigos de programação previamente redigidos e salvos em arquivo com extensão R (os scripts) e arquivos de texto. Na janela superior, à direita, a primeira aba gerencia diferentes áreas de trabalho. Já a segunda aba registra o histórico de todos os scripts, funções e ações executadas. Na parte inferior, à esquerda, localiza-se a janela do Console, que é a mesma janela padrão disponibilizada no R. Na janela inferior, à direita, são agrupadas outras 4 abas: a primeira delas é um gerenciador de arquivos (aba File); na segunda, são exibidos os gráficos gerados pelo RStudio (aba Plots); na terceira, são apresentados os pacotes já instalados (aba Packages) e a quarta aba trata-se da Ajuda (aba Help) do R/RStudio. 
Figura 1 - Interface RStudio

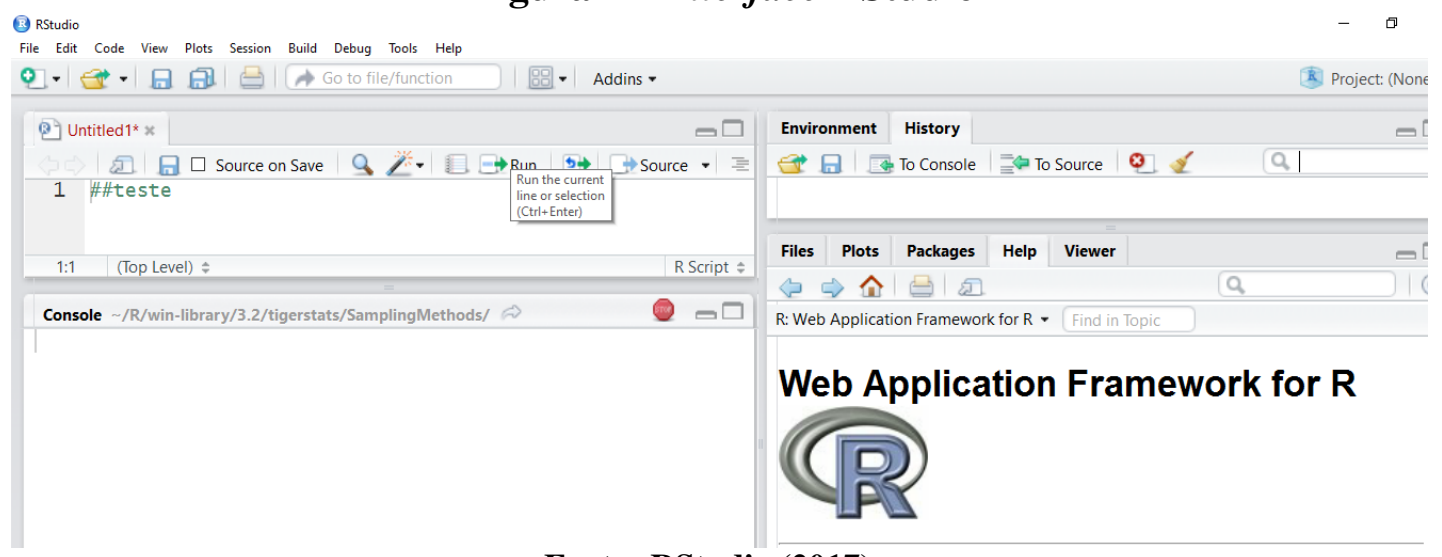

Fonte: RStudio (2017).

\subsection{Shiny}

A partir do RStudio, uma das formas desenvolvidas pela comunidade de programadores para viabilizar o uso de R diretamente na Internet foi o pacote Shiny (CHANG et al., 2017), até então com poucas aplicações relatadas na literatura nacional (KONRATH et al., 2013; HENNING et al., 2016; MÜLLER; ZABALA, 2016). Com esse pacote, é possível transformar análises complexas em aplicações interativas e amigáveis utilizadas via web.

Então, o software R não serve apenas para análises quantitativas; com ele também se pode construir aplicações interativas na web (RADU et al., 2014; BEELEY, 2016) por meio da utilização de pacotes como o Shiny. O pacote Shiny facilita a criação de aplicações interativas na $w e b$ que podem ser utilizadas na apresentação de dados de maneira interativa com inovadores recursos de visualização. Além disso, oferece uma interessante interface gráfica para disponibilizar aplicações para as pessoas que não têm familiaridade com R.

Desse modo, diversas aplicações podem ser desenvolvidas com o Shiny. Por exemplo, é possível desenvolver aplicações de valores padrão para uma classe de elementos (default CSS) totalmente escritas em R, páginas na web (por exemplo, linguagem de marcação de hipertexto (HTML), folhas de estilo em cascata (CSS), linguagem orientada a objetos (JavaScript) e biblioteca de funções JavaScript que interage com o HTML (jQuery) e documentos interativos que contêm janelas, botões, ícones, menus, barras de rolagem e outras funcionalidades (widgets) Shiny incorporados (BEELEY, 2016).

No desenvolvimento de aplicativos web, o Shiny apresenta dois scripts que são salvos em diretório do R. O primeiro script, ui.R, apresentado no Quadro 1, é referente à interface do usuário (UI). A UI tem a função de controlar o layout e a aparência do aplicativo. 


\section{Quadro 1 - Estrutura simples do UI}

\begin{tabular}{|l|l|}
\hline Função & Finalidade \\
\hline library(shiny) & Carregar o pacote Shiny. \\
\hline shinyUI(fluidPage) & Criar uma interface com o usuário. \\
\hline titlePanel() & Criar um painel contendo um título do aplicativo. \\
\hline WsidebarLayout & $\begin{array}{l}\text { Criar um layout com uma barra lateral e área princi- } \\
\text { pal. A barra lateral é exibida com uma cor de fundo } \\
\text { distinta e geralmente contém controles de entrada. A } \\
\text { área principal ocupa } 2 / 3 \text { da largura horizontal e geral- } \\
\text { mente contém saídas. }\end{array}$ \\
\hline sidebarPanel() & $\begin{array}{l}\text { Criar um painel com barra lateral, que contenha con- } \\
\text { troles de entrada que, por sua vez, possam ser passa- } \\
\text { dos para SidebarLayout. }\end{array}$ \\
\hline mainPanel() & $\begin{array}{l}\text { Criar um painel principal contendo elementos de } \\
\text { saída que, por sua vez, pode ser passado para side- } \\
\text { barLayout. }\end{array}$ \\
\hline
\end{tabular}

Fonte: Adaptado de Chang et al. (2017).

O segundo scripts é o servidor e recebe o nome de server.R. A estrutura simples do server pode ser visualizada no Quadro 2.

Quadro 2 - Estrutura simples do server

\begin{tabular}{|l|l|}
\hline Função & Finalidade \\
\hline library(shiny) & Carregar o pacote Shiny. \\
\hline shinyServer() & $\begin{array}{l}\text { Definir a lógica do servidor do aplicativo Shiny. Isso } \\
\text { geralmente envolve a criação de funções que ma- } \\
\text { peiam entradas de usuários para vários tipos de saída. }\end{array}$ \\
\hline function(input,output) \{\} & Funções render (funções do R) \\
\hline
\end{tabular}

Fonte: Adaptado de Chang et al. (2017).

Para o painel de opções laterais, há um conjunto de dispositivos de controle de entrada, que podem ser utilizados, conforme apresentado no Quadro 3. Como exemplo, lista de seleção, botão para escolher um ou vários itens de uma lista de valores, campo para inserção de novos valores são opções para atribuição de valores de entrada em uma variável input. 
Quadro 3 - Conjunto de dispositivos de controle de entrada

\begin{tabular}{|c|c|}
\hline Função & Finalidade \\
\hline ActionButton & $\begin{array}{l}\text { Criar um botão de ação, cujo valor é inicialmente zero } \\
\text { e aumenta em um cada vez que é pressionado. }\end{array}$ \\
\hline actionLink & $\begin{array}{l}\text { Criar um link de ação cujo valor é, inicialmente, zero } \\
\text { e aumenta em um cada vez que é pressionado. }\end{array}$ \\
\hline checkboxGroupInput & $\begin{array}{l}\text { Criar um grupo de caixas de seleção que podem ser } \\
\text { usadas para alternar várias opções de modo indepen- } \\
\text { dente. }\end{array}$ \\
\hline checkboxInput & $\begin{array}{l}\text { Criar uma caixa de seleção que pode ser usada para } \\
\text { especificar valores lógicos. }\end{array}$ \\
\hline dateInput & $\begin{array}{l}\text { Criar uma entrada de texto que, quando clicada, traz } \\
\text { um calendário no qual o usuário pode clicar para se- } \\
\text { lecionar datas. }\end{array}$ \\
\hline dateRangeInput & $\begin{array}{l}\text { Criar um par de entradas de texto que, quando cli- } \\
\text { cadas, trazem calendários nos quais o usuário pode } \\
\text { clicar para selecionar datas. }\end{array}$ \\
\hline fileInput & $\begin{array}{l}\text { Criar um controle de upload de arquivos que pode ser } \\
\text { usado para carregar um ou mais arquivos. }\end{array}$ \\
\hline numericInput & $\begin{array}{l}\text { Criar um controle de entrada para a entrada de valores } \\
\text { numéricos. }\end{array}$ \\
\hline passwordInput & Criar um controle de senha para a entrada de senhas. \\
\hline radioButtons & $\begin{array}{l}\text { Criar um conjunto de botões usados para selecionar } \\
\text { um item de uma lista. }\end{array}$ \\
\hline selectInput & $\begin{array}{l}\text { Criar uma lista de seleção que pode ser usada para } \\
\text { escolher um único ou vários itens de uma lista de va- } \\
\text { lores. }\end{array}$ \\
\hline sliderInput & $\begin{array}{l}\text { Construir um widget de controle deslizante para sele- } \\
\text { cionar um valor numérico de um intervalo. }\end{array}$ \\
\hline submitButton & $\begin{array}{l}\text { Criar um botão de enviar para um aplicativo. Apli- } \\
\text { cativos que incluem um botão de enviar não atuali- } \\
\text { zam automaticamente suas saídas quando as entradas } \\
\text { são alteradas. É esperado até que o usuário clique } \\
\text { explicitamente no botão enviar. O uso do submitBut- } \\
\text { ton,geralmente, é desencorajado em favor do Action- } \\
\text { Button mais versátil. }\end{array}$ \\
\hline textInput & $\begin{array}{l}\text { Criar um controle de entrada para a entrada de valores } \\
\text { de texto não estruturados. }\end{array}$ \\
\hline
\end{tabular}

Fonte: Adaptado de Chang et al. (2017).

Para criar a relação entre a interface do usuário e o server, um conjunto de funções render (guarda resultados do r) e output (mostra os resultados na interface de usuário) trabalham juntas para apresentar a saída do R, conforme Quadro 4. Assim, para um novo aplicativo no Shiny, cria-se um diretório no R e salvam-se os dois scripts (ui.R; server.R) dentro desse diretório. Para executar o aplicativo, clica-se no botão runApp ou digita-se o comando runApp() com o nome do diretório. 
Quadro 4 - Conjunto de dispositivos de saída

\begin{tabular}{|c|c|c|}
\hline \multicolumn{2}{|r|}{ Função } & Finalidade \\
\hline \multirow{7}{*}{ render() } & renderDataTable & $\begin{array}{l}\text { Criar uma versão reativa de uma dada função que } \\
\text { retorna um dataframe (ou matriz), que será renderi- } \\
\text { zada com a biblioteca DataTables. }\end{array}$ \\
\hline & renderImage & $\begin{array}{l}\text { Renderizar uma imagem reativa que é adequada } \\
\text { para atribuir a um slot de saída. }\end{array}$ \\
\hline & renderPlot & $\begin{array}{l}\text { Renderizar um gráfico reativo que é adequado para } \\
\text { atribuir a um slot de saída. }\end{array}$ \\
\hline & renderPrint & $\begin{array}{l}\text { Criar uma versão reativa da função dada que captura } \\
\text { qualquer saída impressa. }\end{array}$ \\
\hline & renderTable & $\begin{array}{l}\text { Criar uma tabela reativa que é adequada para atri- } \\
\text { buir a um slot de saída. }\end{array}$ \\
\hline & renderText & $\begin{array}{l}\text { Criar uma versão reativa de uma dada função, que } \\
\text { também usa o cat para transformar seu resultado em } \\
\text { um vetor de caracteres de um único elemento. }\end{array}$ \\
\hline & renderUI & $\begin{array}{l}\text { Criar uma versão reativa de uma função que gera } \\
\text { HTML usando a biblioteca Shiny UI. }\end{array}$ \\
\hline \multirow{7}{*}{ output() } & dataTableOutput & $\begin{array}{l}\text { Renderizar uma renderTable ou renderDataTable } \\
\text { dentro de uma página do aplicativo. A renderTa- } \\
\text { ble usa uma tabela HTML padrão, enquanto a ren- } \\
\text { derDataTable usa a biblioteca JavaScript DataTables } \\
\text { para criar uma tabela interativa com mais recursos. }\end{array}$ \\
\hline & imageOutput & $\begin{array}{l}\text { Renderizar um renderPlot ou renderImage dentro de } \\
\text { uma página do aplicativo. }\end{array}$ \\
\hline & plotOutput & $\begin{array}{l}\text { Renderizar um renderPlot ou renderImage dentro de } \\
\text { uma página do aplicativo. }\end{array}$ \\
\hline & verbatimTextOutput & $\begin{array}{l}\text { Renderizar uma variável de saída reativa como texto } \\
\text { dentro de uma página de aplicativo. }\end{array}$ \\
\hline & tableOutput & $\begin{array}{l}\text { Renderizar uma renderTable ou renderDataTable } \\
\text { dentro de uma página do aplicativo. O renderTa- } \\
\text { ble usa uma tabela HTML padrão, enquanto o ren- } \\
\text { derDataTable usa a biblioteca JavaScript DataTables } \\
\text { para criar uma tabela interativa com mais recursos. }\end{array}$ \\
\hline & textOutput & $\begin{array}{l}\text { Renderizar uma variável de saída reativa como texto } \\
\text { dentro de uma página de aplicativo. O texto será } \\
\text { incluído dentro de uma tag HTML div por padrão. }\end{array}$ \\
\hline & UiOutput \& htmlOutput & $\begin{array}{l}\text { Renderizar uma variável de saída reativa como } \\
\text { HTML dentro de uma página de aplicativo. O texto } \\
\text { será incluído em uma tag HTML. }\end{array}$ \\
\hline
\end{tabular}

Fonte: Adaptado de Chang et al. (2017).

Na Figura 2, é apresentado um exemplo de um ui.R do Shiny que constrói um histograma, permitindo ao usuário modificar o número de classes de forma interativa. 
Figura 2 - Arquivo ui.R do exemplo para construção de um histograma (B) RStudio

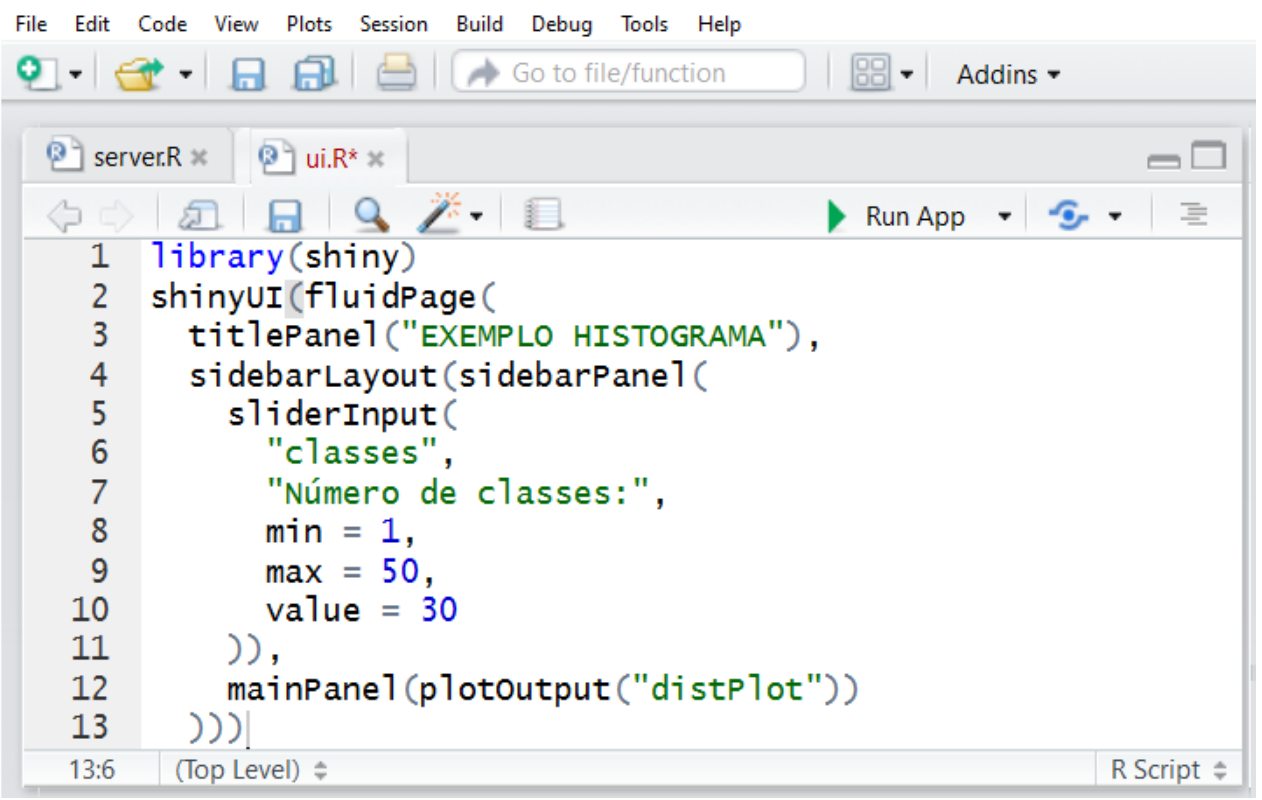

Fonte: Elaborada pelos autores.

Na Figura 3, é apresentado o arquivo server.R., para a criação de um histograma.

Figura 3 - Arquivo server.R

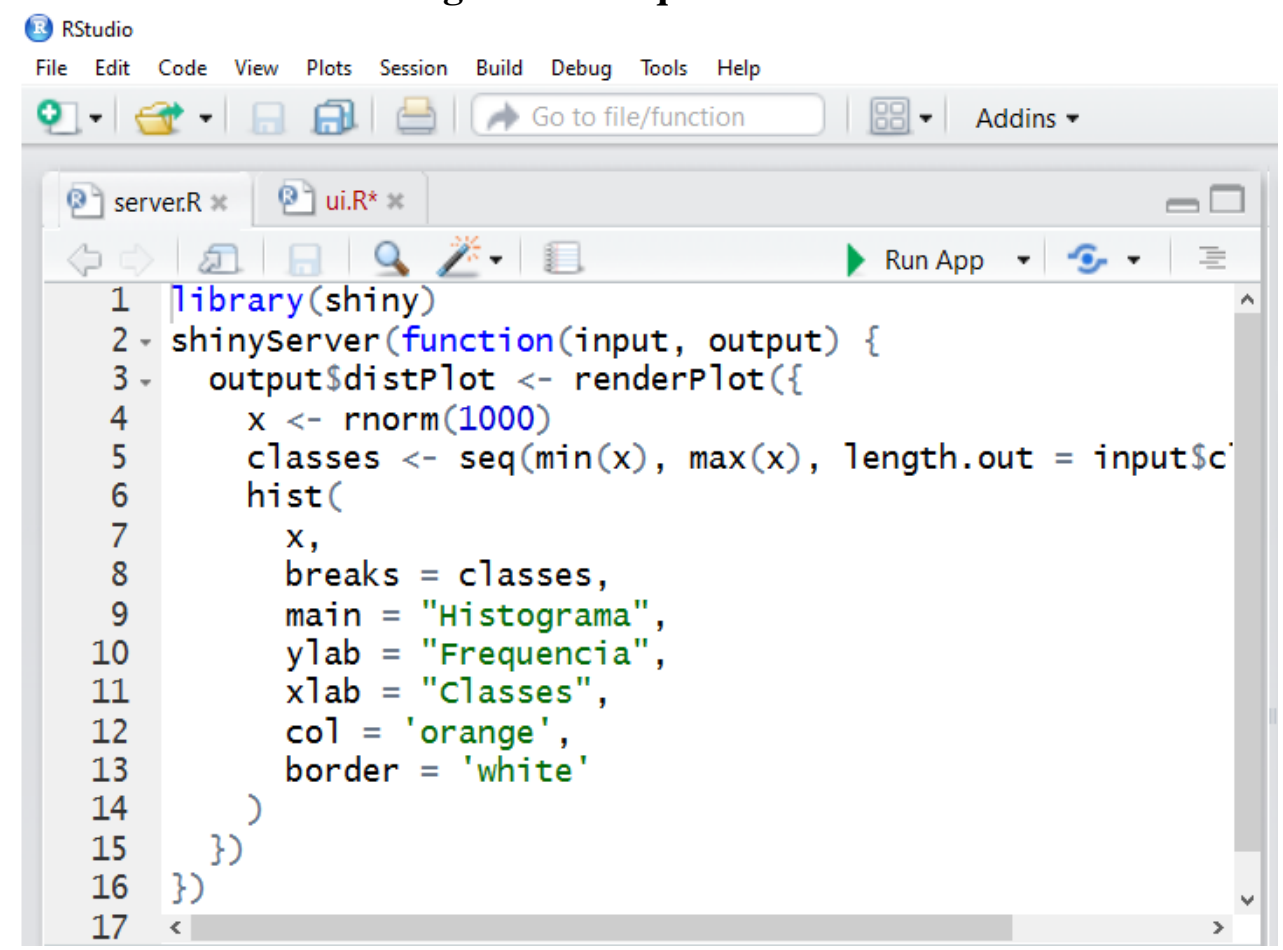

Fonte: Elaborada pelos autores. 
$\mathrm{Na}$ Figura 4, é apresentado o resultado da aplicação.

Figura 4 - Exemplo de app no Shiny

\section{EXEMPLO HISTOGRAMA}

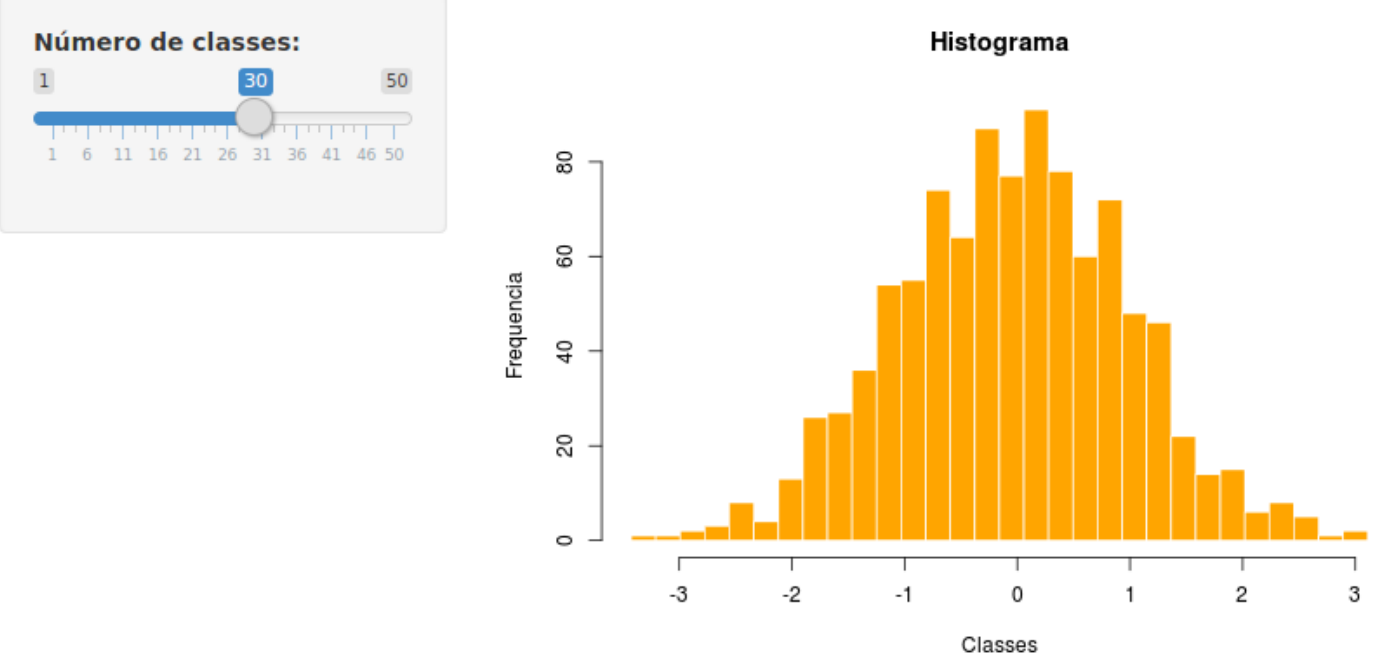

Fonte: Elaborada pelos autores.

\section{APLICATIVOS WEB DO SHINY NO ENSINO E NA PESQUISA}

Por conta das características de interatividade e de visualização, o desenvolvimento e a utilização de aplicativos web contribuem de forma positiva no ensino e na aprendizagem de conteúdos de Estatística (WILLIAMS; WILLIAMS, 2018). Sendo o Shiny um pacote do R, que permite o desenvolvimento de aplicativos que podem ser manipulados pela Internet, torna-se uma alternativa promissora para contribuir com inovações no ensino de Estatística. É importante ressaltar que, nesse caso, o mesmo software que é aplicado nas análises serve também para a geração dos aplicativos web. Uma vez construídas, as análises podem ser compartilhadas e discutidas entre estudantes e professores, ampliando as possibilidades de interação e de debates sobre os dados envolvidos. Além disso, seu uso possibilita que sejam ampliadas as práticas de simulação computacional.

A elaboração de documentos dinâmicos, tais como os aplicativos web, pode auxiliar na reprodutibilidade de pesquisas (XIE, 2015; BAUMER; UDWIN, 2015). Esses documentos interativos podem ser desenvolvidos com o Shiny, usando o RMarkdown (ALLAIRE et al., 2016a) no RStudio. O R Markdown é um sistema de uso fácil que permite combinar a análise estatística com a produção de um documento, ou seja, processar um script do R e gerar um documento com gráficos e texto estilizado (BAUMER et al., 2014). Aos que não são usuários do RStudio, é possível elaborar aplicações com RMarkdown e Shiny no Pandoc1 (BEELEY, 2016).

Na Figura 5, é apresentado um aplicativo que foi construído em conjunto com alguns pacotes do R, RMarkdown, Shiny, leaflet (CHENG et al., 2016), flexdashsboard (ALLAIRE et al., 2016b), plotly (SIEVERT et al., 2016) para visualização das notas do Exame Nacional do 
Ensino Médio (ENEM) no Estado de Santa Catarina.

Há pesquisas que relatam o desenvolvimento de aplicações de Shiny para outras áreas, além das voltadas à Estatística, tais como aplicações educacionais de mineração de dados em classificação de textos (NUNZIO, 2016) e aprendizagem automática ou de máquina (machine learning) (NUNZIO, 2016; NUNZIO et al., 2016).

\subsection{Aplicativo das notas do ENEM no Estado de Santa Catarina}

Um exemplo de aplicação desenvolvido por meio do pacote Shiny foi o aplicativo para visualização de notas do ENEM no Estado de Santa Catarina, disponibilizado no seguinte link: https://silvio.shinyapps.io/ENEMSC/. O mesmo possui diversas funções que são descritas, conforme a numeração apresentada na Figura 5.

\section{Figura 5 - Aplicativo construído para visualização das notas do ENEM no Estado de Santa Catarina}

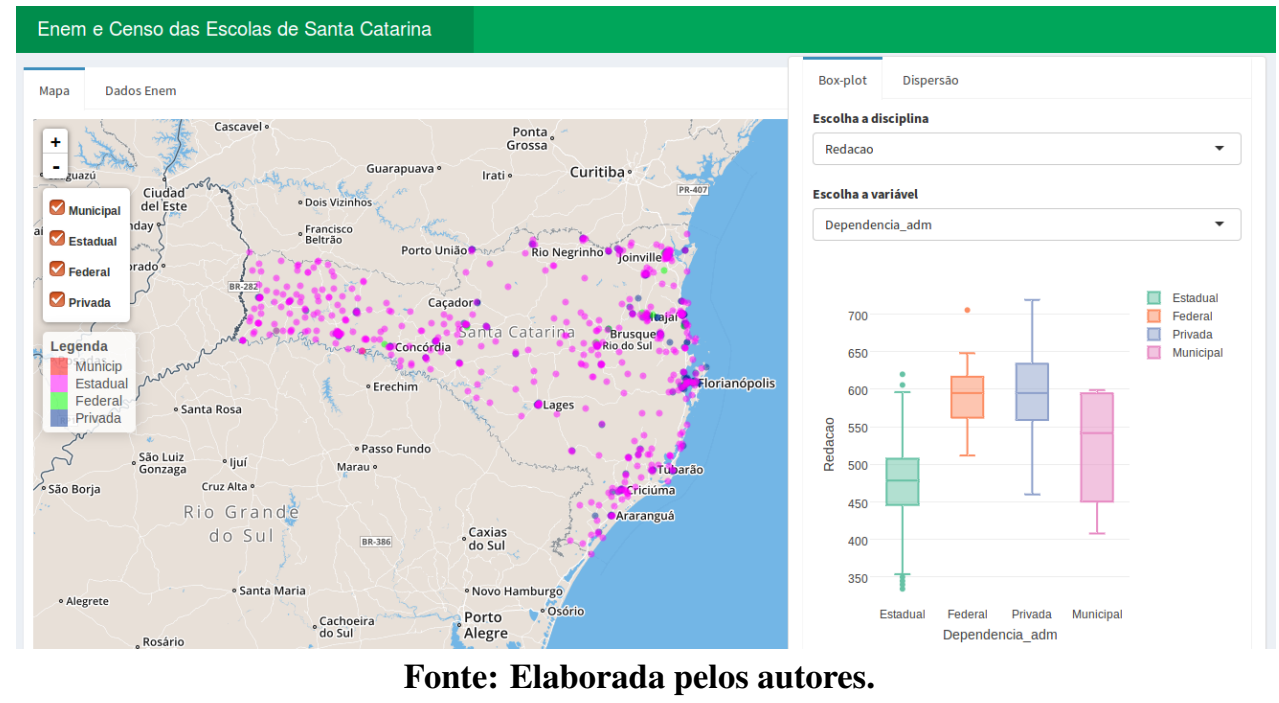

1 - Título: O nome da página é "Enem e Censo das Escolas de Santa Catarina". A proposta é ter uma página dinâmica na qual o usuário tenha condições de fazer as suas análises dos dados apresentados. A página está em constante construção, pois a proposta é que os dados sejam atualizados todos os anos, do mesmo modo em que serão incluídos os dados referentes ao censo educacional para análise futura.

2 - Link Mapa e Dados Enem: Clicando no link dados ENEM, abre-se o banco de dados utilizado para construção da página. Clicando no link Mapa, ele retorna à página inicial, com o mapa do Estado de Santa Catarina.

3 - Botão para aproximar e distanciar o Mapa: Aumentando o foco no nome de determinada cidade, obtém-se uma visão ampliada de escolas que realizaram o ENEM. Pode-se também observar as diferenças em relação ao tamanho do "círculo-rosa" que identifica a escola, sendo que o tamanho faz referência à quantidade de alunos daquela escola que realizaram as provas do ENEM. 
4 - Seleção por tipo de escola: Nesse link, o usuário pode selecionar o tipo de escola, de acordo com a dependência administrativa, a qual pode ser: municipal, estadual, federal e privada.

5 - Legenda: Legenda por tipo de dependência administrativa da escola.

6 - Link Gráfico Box-plot e Gráfico de Dispersão: Clicando no link Dispersão, abrese o Gráfico de dispersão e a seleção de variáveis para construção do gráfico. Ao clicar no link Box-plot, retorna-se à página inicial com o gráfico de box-plot e sua respectiva caixa de seleção.

7 - Caixa de seleção por tipo de disciplina: Nesse link, o usuário pode escolher a disciplina da prova aplicada no ENEM: Linguagem, Redação, Matemática, Humanas e Natureza (variáveis contínuas).

8 - Caixa de seleção por tipo de variável: O usuário pode selecionar a variável (variáveis categóricas) que deseja relacionar com as notas do ENEM. As variáveis são: dependência administrativa; localização; porte da escola; indicador de permanência na escola; indicador de nível socioeconômico; faixa de indicador de formação docente.

9 - Seleção no gráfico box-plot: É possível selecionar no próprio gráfico box-plot e assim efetuar comparações. As variáveis também podem ser selecionadas no próprio gráfico para fazer comparações.

10 - Caixa de seleção por tipo de variável no eixo x: Nesse link, é possível selecionar a variável independente (variáveis contínuas) para a análise: número de alunos; números de participantes no ENEM; taxa de participação; indicador de formação docente; taxa de aprovação; taxa de reprovação; taxa de abandono

11 - Coeficiente de correlação: Nesse link, obtém-se o coeficiente de correlação referente à disciplina escolhida e ao eixo da variável x, conforme item 10.

Na Figura 6, é apresentado o gráfico de dispersão e as variáveis selecionadas, conforme descritos nos item 10 e 11. Os dados utilizados para a construção do aplicativo estão disponíveis para download no portal do Instituto Nacional de Estudos e Pesquisas Educacionais Anísio Teixeira (INEP - Instituto Nacional de Estudos e Pesquisas Educacionais Anísio Teixeira., 2017). 


\section{Figura 6 - Gráfico de dispersão das variáveis notas do Enem e variáveis selecionadas}

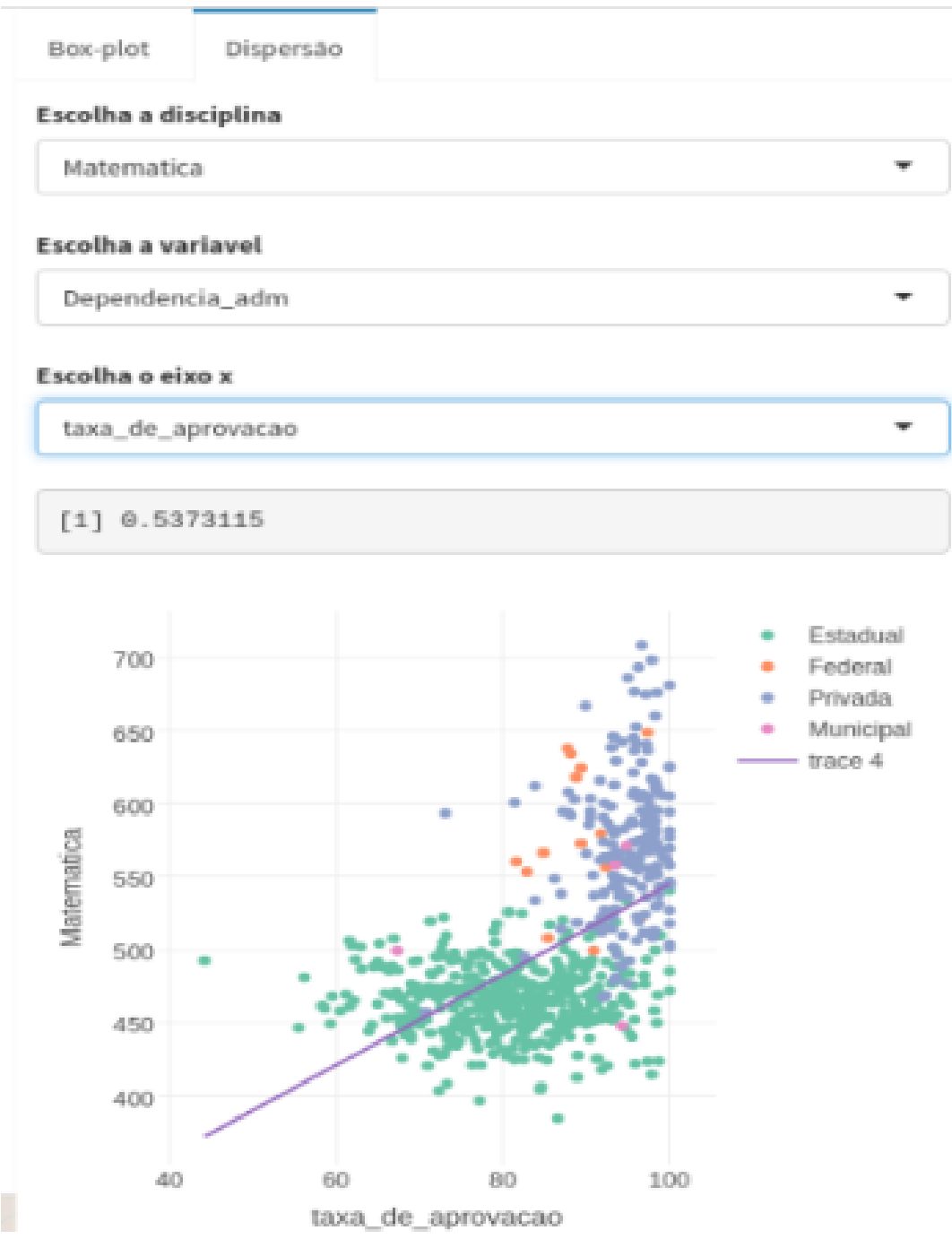

Fonte: Elaborada pelos autores.

Em relação ao aplicativo desenvolvido, pode-se verificar que o mesmo envolve conceitos estatísticos, relacionados com a associação entre variáveis, como o coeficiente de correlação e os gráficos de dispersão e boxplot. São conceitos simples, mas com o aplicativo desenvolvido, o processo de ensino e de aprendizagem pode se tornar mais interativo e dinâmico. Além disso, pode-se dizer que o aplicativo é inovador, pois o pesquisador/aluno tem a informação apresentada na tela de forma instantânea, à medida que altera os parâmetros dos gráficos. Essa interatividade ocorre sem a necessidade de alterar os scripts gerados pelo Shiny, ou seja, não é necessário conhecimento de linguagem de programação $\mathrm{R}$ para uso do aplicativo, apenas para construí-lo.

O uso do Shiny no processo de ensino e de aprendizagem apresenta oportunidades e, é claro, desafios. Um aspecto positivo é a flexibilidade que possibilita uma abordagem híbrida de ensino (PIMMER et al., 2016), e permite ao professor a disposição ad hoc e post hoc de materiais de aula e suplementares. Os estudantes, de modo individual ou colaborativo, ainda 
podem desenvolver suas próprias formas de representação das análises, seja no aspecto visual ou textual. Todas essas etapas podem ocorrer em espaços dentro e fora da sala de aula, uma vez que os alunos podem usar os aplicativos em dispositivos móveis, como tablets e smartphones, de modo interativo e integrado.

Por outro lado, os desafios são os mesmos de todas as inovações no ensino, quer sejam tecnológicas ou não. Os autores desse trabalho concordam com Gan, Menkhoff e Smith (2015) que questionam como abordagens tecnológicas interativas podem efetivamente ser integradas no planejamento de cursos e aulas. Do mesmo modo, a aplicação efetiva é um desafio. O Shiny, assim como outras tecnologias interativas e aplicações para mobile learning, representa uma oportunidade para contínuo aprendizado do professor e apresenta qualidades que podem contribuir para a aprendizagem no Ensino Superior na e além da sala de aula. Nesse sentido, é fundamental investigar a efetividade dos demais aspectos destas aplicações (GAN et al., 2015).

No âmbito da realização de atividades de pesquisa, o Shiny apresenta-se como uma importante ferramenta de apoio e, principalmente, de reprodutibilidade. A partir do R, Rstudio e Shiny, pode-se orientar pesquisadores na criação e no desenvolvimento de objetos de aprendizagem que possibilite a realização de investigações dinâmicas e reprodutíveis, pois tais programas apresentam ferramentas práticas para a coleta de dados, análise de dados e apresentação dos resultados (GANDRUD, 2013). Concordando com Gandrud (2013), tem-se, assim, a perspectiva da formação de pesquisadores engajados na viabilidade da reprodutibilidade das pesquisas.

\section{CONSIDERAÇÕES FINAIS}

Este artigo explorou as características e as potencialidades do pacote Shiny do software $\mathrm{R}$ para o desenvolvimento de um aplicativo web voltado ao ensino e à pesquisa. Entende-se que as TIC que apoiam diversas tarefas do cotidiano estão também presentes nos diversos ambientes educacionais. Quando inseridas no processo de ensino e de aprendizagem, viabilizam a construção de novos modos de ensinar e de aprender. Nesse cenário, é fundamental que o professor, como mediador da construção de conhecimento, saiba aproveitar esses recursos, fazendo com que eles auxiliem no desenvolvimento de práticas docentes inovadoras. Com relação ao ensino de Estatística, objeto de muitos currículos dos cursos de Ensino Superior, as TIC estão cada vez mais presentes, incentivando e proporcionando o uso de software que permita a realização de atividades mais dinâmicas e interativas se comparadas às análises feitas somente nos ambientes lápis/papel ou giz/quadro. Nesse contexto, a utilização de software livre é uma alternativa viável e promissora. A inserção e o estímulo à utilização de software livre em Estatística, como o R, contribuem para a democratização e a melhora do processo de ensino e de aprendizagem dessa disciplina. R é uma linguagem e um ambiente para computação estatística e por se tratar de uma linguagem de programação, pode haver certa resistência dos alunos ou profissionais, que podem mostrar preferência por um programa com interface mais amigável. Existem diversos pacotes que podem fornecer uma interface amigável, dentre os quais podemos destacar o 
pacote Shiny, que permite desenvolver aplicativos web com representação das análises, tanto no aspecto visual quanto textual. Além disso, pode ser utilizado em dispositivos móveis, como tablets e smartphones, de modo interativo e integrado, o que amplia os espaços e tempos de ensino e aprendizagem.

Neste artigo, foram apresentadas algumas das possibilidades que o pacote Shiny pode proporcionar tanto ao ensino quanto à pesquisa. A partir da análise da experiência com as práticas aqui descritas, acredita-se que o Shiny pode ser utilizado em processo de ensino e de aprendizagem de Estatística e em atividades de pesquisa com o objetivo de atingir melhores resultados em cada um desses contextos. Os autores continuam o trabalho nessa área, com o desenvolvimento de dois aplicativos utilizando o Shiny, um na área de Controle Estatístico de Processos, que pode ser acessado no link: https://r-nnq.shinyapps.io/index; e outro na área de Previsão, que pode ser acessado em: https://estatistica.inf.ufsc.br/nnq/forecast. Espera-se, assim, ampliar os espaços de discussão e de utilização de alternativas inovadoras para o ensino de Estatística e para a universalização da pesquisa em diferentes áreas. 


\section{REFERÊNCIAS}

ALLAIRE, J. J. et al. Rmarkdown: Dynamic Documents for R. 2016. Disponível em: <https: //CRAN.R-project.org/package=rmarkdown>. Acesso em: 02 out. 2016.

ALLAIRE, J. J. et al. Flexdashboard: R Markdown Format for Flexible Dashboards. 2016. Disponível em: <https://cran.r-project.org/package=flexdashboard>. Acesso em: 03 out. 2016.

BAIER, T.; NEUWIRTH, E. Excel:: Com::r. computational statistics. Computational Statistics, Springer, v. 22, n. 1, p. 91-108, 2007.

BAUMER, B. et al. R Markdown: Integrating a reproducible analysis tool into introductory statistics. arXiv preprint arXiv:1402.1894, 2014.

BAUMER, B.; UDWIN, D. R markdown. Wiley Interdisciplinary Reviews: Computational Statistics, Wiley Online Library, v. 7, n. 3, p. 167-177, 2015.

BEELEY, C. Web application development with R using Shiny. Birmingham, UK: Packt Publishing Ltd, 2016.

CAMPOS, R. R.; CAZARINI, E. W. Sistemas ERP de Código Aberto: uma opção para as pequenas indústrias? In: International Conference on Information Systems and Technology Management. São Paulo, SP: Congresso Internacional de Gestão da Tecnologia e Sistemas de Informação, 2005. v. 2.

CHANG, W. et al. Shiny: Web Application Framework for R. 2017. Disponível em: <https: //CRAN.R-project.org/package=shiny>. Acesso em: 10 mar. 2017.

CHENG, J. et al. Leaflet: Create Interactive Web Maps with the JavaScript 'Leaflet'. Library. 2016. Disponível em: <https://CRAN.R-project.org/package=leaflet>. Acesso em: 03 out. 2016.

CORREA, J.; SPINOLA, M. Adoção, seleção e implantação de um ERP livre. Production, SciELO Brasil, v. 25, n. 4, p. 956-970, 2015.

FERNANDES, G. C. et al. Um comparativo dos paradigmas de software livre e proprietários no contexto do mercado de trabalho e das universidades. In: Anais do Congresso Nacional Universidade, EAD e Software Livre. Belo Horizonte, MG: [s.n.], 2012. v. 3, n. 2, p. 1-4.

FOX, J. Extending the r commander by "plug-in" packages. R News, v. 7, n. 3, p. 46-52, 2007.

GAN, B.; MENKHOFF, T.; SMITH, R. Enhancing students' learning process through interactive digital media: New opportunities for collaborative learning. Computers in Human Behavior, Elsevier, v. 51, p. 652-663, 2015.

GANDRUD, C. Reproducible research with $\mathbf{R}$ and $\mathbf{R}$ studio. Boca Raton, Florida: CRC Press, 2013.

GOULD, R. Statistics and the modern student. International Statistical Review, Wiley Online Library, v. 78, n. 2, p. 297-315, 2010.

HENNING, E. et al. para além da computação estatística: o uso do ambiente R para o ensino de métodos numéricos. RENOTE, v. 14, n. 1, 2016. 
INEP - Instituto Nacional de Estudos e Pesquisas Educacionais Anísio Teixeira. Inep divulga os microdados do Enem 2016. 2017. Disponível em: < http://portal.inep.gov.br/artigo/-/asset_ publisher/B4AQV9zFY7Bv/content/inep-divulga-os-microdados-do-enem-2016/21206>.

Acesso em: 03 out. 2017.

KATAOKA, V. Y. et al. O uso do r no ensino de probabilidade na educação básica: Animation e teachingdemos (the use of $r$ in probability teaching at basic education: Animation and teaching demos). Proc. $1^{\circ}$ Simpósio Nacional de Probabilidade e Estatística, São Paulo, 2008.

KONRATH, A. C. et al. Applications in teaching Statistical Quality Control with different R interfaces. In: IEEE. Global Engineering Education Conference (EDUCON). Berlim, 2013. p. $146-155$.

MINUTO, A.; PITTARELLO, F.; NIJHOLT, A. Smart material interfaces for education. Journal of Visual Languages \& Computing, Elsevier, v. 31, p. 267-274, 2015.

MÜLLER, Thaísa Jacintho; ZABALA, Filipe Jaeger. Avaliação e correção automática no software livre RStudio. RENOTE, v. 14, n. 1, 2016.

NUNZIO, G. M. Can you learn it?: Probably! Developing Learning Analytics Tools in R. In: Proceedings of the 16th ACM/IEEE-CS on Joint Conference on Digital Libraries. Newark, NJ: ACM, 2016. p. 213-214.

NUNZIO, G. M.; MAISTRO, M.; ZILIO, D. Gamification for machine learning: The classification game. medicine, v. 9, p. 2, 2016.

PIMMER, C.; MATEESCU, M.; GRÖHBIEL, U. Mobile and ubiquitous learning in higher education settings. a systematic review of empirical studies. Computers in Human Behavior, Elsevier, v. 63, p. 490-501, 2016.

R CORE TEAM. R: A language and environment for statistical computing. 2017. Disponível em: <http://www.R-project.org/>. Acesso em: 03 fev. 2017.

RACINE, Jeffrey S. Rstudio: A Platform-Independent IDE for R and Sweave. Journal of Applied Econometrics, Wiley Online Library, v. 27, n. 1, p. 167-172, 2012.

RADU, M. et al. Using R To Get Value Out Of Public Data. Romanian Statistical Review, v. 62, n. 2, p. 109-128, 2014.

SCHUYTEN, G.; THAS, O. Statistical thinking in computer-based learning environments. International Statistical Review, Wiley Online Library, v. 75, n. 3, p. 365-371, 2007.

SIEVERT, C. et al. Plotly: Create Interactive Web Graphics via 'plotly.js'. 2016. Disponível em: <https://CRAN.R-project.org/package=plotly.> Acesso em: 03 fev. 2016.

VERZANI, J. Using $\mathrm{r}$ in introductory statistics courses with the pmg graphical user interface. Journal of Statistics Education, Taylor \& Francis, v. 16, n. 1, 2008.

WILLIAMS, I. J.; WILLIAMS, K. K. Using an R shiny to enhance the learning experience of confidence intervals. Teaching Statistics, Wiley Online Library, v. 40, n. 1, p. 24-28, 2018.

XIE, Y. Dynamic Documents with R and knitr. Boca Raton, Florida: CRC Press, 2015. 